\title{
HIGH FREQUENCY OF FIRST-LINE ANTI-TUBERCULOSIS DRUG RE- SISTANCE AMONG PERSONS WITH CHRONIC PULMONARY TU- BERCULOSIS AT A TEACHING HOSPITAL CHEST CLINIC
}

\author{
A. FORSON ${ }^{1,2}$, S. KUDZAWU $^{1}$, A. KWARA ${ }^{3}$ and T. FLANIGAN ${ }^{3}$ \\ ${ }^{1}$ Chest Clinic, Korle-Bu Teaching Hospital, ${ }^{2}$ Department of Medicine and Therapeutics, University of Ghana \\ Medical School, ${ }^{3}$ Department of Medicine, Warren Alpert Medical School of Brown University, Rhode Is- \\ land, USA
}

Corresponding Author: Dr Audrey Forson

Conflict of interest: None declared

\section{SUMMARY}

Introduction: The burden of MDR-TB is unknown in areas that do not have drug susceptibility testing (DST), but its frequency is expected to be higher in previously treated cases. Where DST is not available the WHO recommended standardized retreatment (Category II) regimen is given to previously treated $\mathrm{TB}$ patients

Objective: To evaluate the frequency and pattern of drug resistance of Mycobacterium tuberculosis isolated from patients with chronic smear positive pulmonary tuberculosis.

Method: We conducted a retrospective review of mycobacterial cultures and drug susceptibility testing (DST) performed on sputum samples collected, between January 2005 and September 2006, from 40 patients with pulmonary TB who had failed at least one standard retreatment regimen. Clinical data was extracted from patients' case notes.

Results: M. tuberculosis was recovered from 28 (70\%) of the 40 patients. Of the 28 culture positive cases, 10 $(36 \%)$ had resistance to at least rifampicin and isoniazid (multi-drug resistant TB), 22 (79\%) isolates had resistance to streptomycin and $13(46 \%)$ to ethambutol. Of the patients with a positive culture, only one $(3.6 \%)$ had a fully susceptible organism. Of the 10 patients with MDR TB, 7 had received two or more retreatment courses.

Conclusion: The frequency of drug resistant TB was high among patients who failed at least one course of category II therapy. Effective combination regimens based on DST is necessary in patients who remain smear positive on the standardized retreatment regimen.

Keywords: drug-resistance, MDR, chronic TB, retreatment regimen.

\section{INTRODUCTION}

Drug resistant tuberculosis (TB), particularly multidrug resistant (MDR) represents a major global public health
Email: agforson@chs.edu.gh

problem. Several studies have found that MDR TB is associated with a higher risk of treatment failure and death. ${ }^{1-6}$ Based on data from 184 countries, it was estimated that $4.3 \%$ of all reported TB cases and $18.5 \%$ of previously treated cases in 2004 were MDR. ${ }^{7} \quad$ In areas where drug susceptibility testing (DST) is not available, a standardized retreatment (category II) regimen is recommended for previously treated TB patients. $^{8}$ This regimen consists of streptomycin (S), rifampicin (R), isoniazid (I), pyrazinamide and ethambutol (E) for two months, followed by one month of the same drugs except for streptomycin and then five months of rifampicin, isoniazid, and ethambutol (2SRHZE-1RHZE-5HRE).

Countries in South America and Sub-Saharan Africa that have well organized DOTS programmes have a prevalence of chronic TB (defined as a case that remains sputum positive at the end of a supervised standard retreatment regimen) is estimated at $<2 \%$. ${ }^{8,9} \mathrm{Ad}-$ herent patients who remain smear positive at the end of the standard retreatment regimen are likely to have MDR TB and would need second-line drugs. In 2006 Green Light Committee (GLC) projects treated less than $5 \%$ of MDR-TB cases that emerged world-wide, and by the end of 2007 only 51 countries had been provided with second-line anti-TB drugs through the GLC. ${ }^{10}$

In areas that do not have second-line drugs, the main option available to persons who have failed a standard treatment regimen is often a repeat of the same Category II regimen. This suboptimal approach to the management of chronic smear positive cases poses a significant transmission risk of resistant TB to close contacts and health care workers who provide care for these patients.

Repeating Category II treatment after failure of a supervised retreatment regimen is likely to increase the 
degree and spectrum of drug resistance to first line drugs.

Patients who had failed at least one previous treatment (the total number of 'retreatment cases') in Ghana in 2006 were 526 (about 5\% of all TB patients), of which the majority were due to relapse/failure and about $10 \%$ of these were defaulters (National TB Program reports). There were 56 patients on retreatment at the Chest Clinic in 2005 and 2006, and chronic TB cases would be a small proportion of this. Outcomes in these patients from Chest Clinic were; about $50 \%$ cure, 12.5 $\%$ failure/relapse, $12.5 \%$ default and $25 \%$ mortality.

The Chest Clinic laboratory in Korle $\mathrm{Bu}$ Teaching Hospital, the tertiary referral hospital for southern Ghana, was the only laboratory under the Ghana Health Service/Ministry of Health performing mycobacterial cultures in the southern half of Ghana in that period. Sufficient information had been sent out by the National TB Program on the availability of the mycobacterial culture service and most DOTS centres in the region referred their chronic patients to Chest clinic for this reason. In this review, we evaluated the frequency and pattern of drug resistance of Mycobacterium tuberculosis isolated from patients with chronic smear positive pulmonary tuberculosis, who remained smear and culture positive for acid-fast bacilli (AFB) at the end of one or more courses of standard retreatment (Category II) therapy.

\section{METHOD}

The culture results of all chronic TB cases (had failed at least one retreatment course) referred to the Korle$\mathrm{Bu}$ Teaching Hospital Chest Clinic laboratory, representing a sample of chronic cases from the south of Ghana were reviewed. As the number of chronic cases in the country was small all patients who fitted the definition of chronic TB were included in the study. A retrospective review was carried out over a period of 21 months, from January 2005 to September 2006, of consecutive patients referred to the Chest Clinic laboratory who had a positive sputum smear test, having failed at least one retreatment course. Patients' case notes were retrieved for information on number of retreatments, HIV status and outcomes. HIV screening, not available in the Chest Clinic, was done at the Public Health Reference Laboratory in Korle Bu.

Counselling and HIV screening of TB patients was selective, based on clinical suspicion of HIV and patients' willingness to be tested. Sputum samples from 33 consecutive patients were received, with six of the 33 patients re-entered into the study as their sputums were re-cultured within the study period, after failing further retreatment courses. Two samples, one spot and an early morning sample, were pooled and pre- treated with $1 \%$ Cetylpyridinium Chloride (CPC) to inhibit the growth of fungi and other bacteria present. All samples were smear positive by Zeihl-Neelsen staining for AFB. Liquefaction and decontamination was carried out with the modified Petroff method. ${ }^{11}$ The specimen was innoculated onto 3 LowensteinJensen (LJ) slopes containing $0.5 \%$ pyruvate, glycerol and PNB (p-nitrobenzoic acid $500 \mathrm{mg} / \mathrm{l}$ ), and were incubated at $37{ }^{\circ} \mathrm{C}$ for up to 8 weeks. Mycobacterium tuberculosis was confirmed by colony morphology, absence of pigment and failure to grow in PNB. Mycobacteria-other than TB (MOTT) were identified by rapid growth within 7 days and growth in $\mathrm{PNB} .^{12}$

Positive $M$. tuberculosis cultures were sub-cultured, and susceptibility testing to isoniazid, rifampicin, streptomycin and ethambutol was performed by the simplified proportion method. ${ }^{13}$ The critical concentrations of the drugs were as follows: isoniazid $0.2 \mathrm{mg} / \mathrm{l}$, rifampicin $40 \mathrm{mg} / \mathrm{l}$, streptomycin $4.0 \mathrm{mg} / \mathrm{l}$, and ethambutol 2.0 $\mathrm{mg} / \mathrm{l}$. The threshold proportion for resistance was $1 \%$ for all drugs. A standard H37Rv strain of known resistance to the drugs tested was used as controls in each batch of tests.

\section{RESULTS}

Forty smear positive sputum samples were cultured of which $28(70.0 \%)$ grew M. tuberculosis, $8(20 \%)$ showed no bacterial growth, 3 had fungal contamination and one grew MOTT (a fast grower). The median age for all patients was 38.8 years with ages ranging between 9 and 75 years. Majority (71.4\%) were male patients and were in the productive age group 25 to 64 years. The characteristics of the 28 patients with positive culture are shown in Table 1.

Table 1 Demographic characteristics of 28 culture positive chronic TB patients

\begin{tabular}{|c|c|}
\hline \multicolumn{2}{|l|}{ CHARACTERISTIC } \\
\hline \multicolumn{2}{|l|}{ Age (yrs) } \\
\hline Mean age & $39.5($ SD 15.5$)$ \\
\hline Median & 38 \\
\hline \multicolumn{2}{|r|}{ Frequency (n) } \\
\hline Male & 20 \\
\hline Female & 8 \\
\hline \multicolumn{2}{|l|}{ Referral site } \\
\hline Chest Clinic \& Accra & 22 \\
\hline Other & 6 \\
\hline \multicolumn{2}{|l|}{ HIV status } \\
\hline Positive & 3 \\
\hline Negative & 7 \\
\hline Not Done & 12 \\
\hline Not Available & 6 \\
\hline
\end{tabular}


Table 2 Frequency of drug resistance among 28 culture positive chronic TB patients

\begin{tabular}{|l|l|l|}
\hline RESISTANCE PATTERN & Number & $\begin{array}{l}\text { Frequen- } \\
\text { cy }\end{array}$ \\
\hline Streptomycin & 22 & $78.57 \%$ \\
\hline MDR (H+R) & 10 & $35.71 \%$ \\
\hline MDR + S & 7 & $25.00 \%$ \\
\hline MDR + E & 6 & $21.43 \%$ \\
\hline Panresistant (to SHR\&E) & 5 & $17.86 \%$ \\
\hline Ethambutol & 13 & $46.43 \%$ \\
\hline H alone & 6 & $21.43 \%$ \\
\hline R alone & 5 & $17.86 \%$ \\
\hline Pansensitive & 1 & $3.57 \%$ \\
\hline Total Resistant TB strains & 28 & \\
\hline
\end{tabular}

Majority of patients were being managed at Chest Clinic, 3 were from other hospitals and clinics in Accra. Six whose records could not be traced were not Chest Clinic patients and had had cultures requested from DOTS centres in Tema and the Central Region, with one from the Ashanti Region.

Data obtained from 22 case notes showed that 6 patients had relapsed after being declared cured, 11 were failures of one or more retreatments, and 8 had a history of default, 3 being recurrent defaulters. The mean number of treatment courses they had had was 2.9, majority having had 2 or more retreatment courses Twelve patients had not had their HIV status determined, 3 others were HIV positive and 7 HIV negative.

The results of DST are shown in Table 2. Of the 28 patients, $10(36 \%)$ had MDR-TB, $6(21 \%)$ had isonia- zid mono resistance and 5 (18\%) had rifampicin mono resistance.

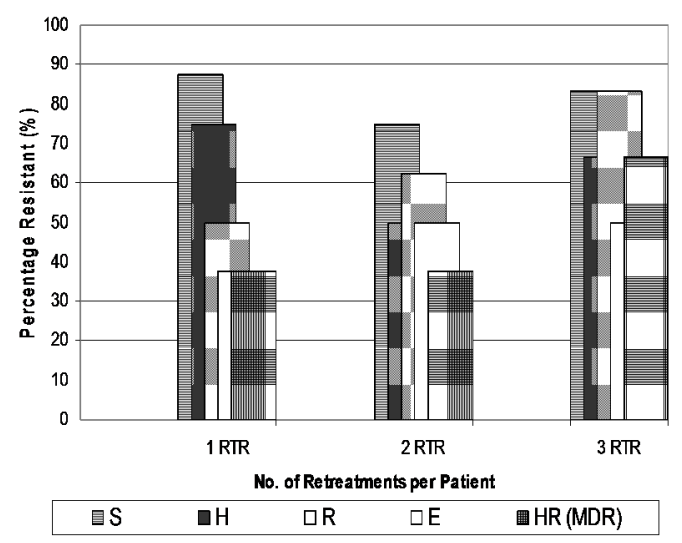

Figure 2 Frequency of Drug Resistant- and MDR- TB in relation to number of retreatment (RTR) regimens

Overall, $79 \%$ had resistance to streptomycin, $18 \%$ had resistance to all four drugs and only one patient (3.6\%) had an isolate susceptible to all 4 drugs. The prevalence of mono- and multiple-drug resistance patterns are shown in a Venn diagram (Figure 1).

Four out of 8 defaulters $(50 \%)$ and 4 out of 6 with relapse-after-cure $(66.6 \%)$ had MDR-TB. The proportions with mono- or multi-drug resistance in relation to the number of retreatments a patient had showed an increasing trend for rifampicin resistance and MDR, though significance could not be determined because of insufficient patient numbers (Figure 2).

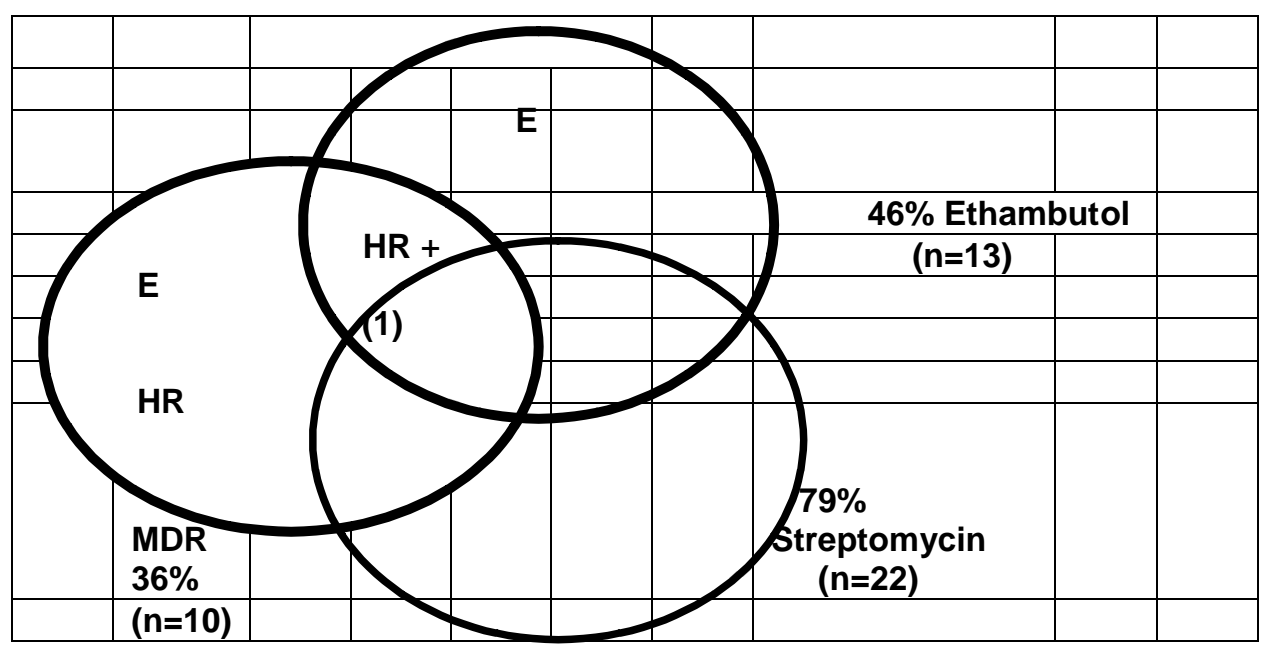

Figure 1 Pattern of Drug Resistance among 28 culture positive patients 



\section{DISCUSSION}

Tuberculosis had a world-wide incidence of 9 million people in 2006, and nearly 1.7 million people die every year from TB. ${ }^{8,14}$ Combination therapy with effective anti-tuberciulosis agents is intended to improve cure rates and prevent the emergence of drug resistance. Resistance to the antituberculosis drugs is being recognized more and more as their effectiveness is subverted by poor adherence, inadequate dosing and prescription of incorrect regimens, effectively exposing organisms to monotherapy. ${ }^{15}$ As developing countries acquire the expertise and the resources required to diagnose it resistant tuberculosis is being recognized more and more.

While a few drug resistance surveys have been carried out on primary TB isolates from treatment-naïve patients in Ghana, only one has studied previously treated patients. Newman MJ et al. in a previous survey of chronic TB patients in this teaching hospital reported a high level of resistance among 18 cultured isolates, with $15(83 \%)$ showing resistance to at least two firstline anti-tuberculosis drugs. ${ }^{16}$ Thirty-three percent (33\%) were MDR which was similar to our review, however only $3.6 \%$ in our review were pan-sensitive to all drugs, compared with $16.7 \%$ in Newman's study, who were likely to respond to further first-line treatment. A well performing DOTS program is the best prevention against chronic disease and MDR-TB. In developing countries that have had a well applied DOTS strategy for several years, chronic cases represent less than $2 \%$ of the total smear-positive pulmonary TB cases. $^{9,10}$

In a Peruvian study on mycobacterial culture and DST of pulmonary TB cases $18 \%$ of cases were MDR-TB, which accounted for $71 \%$ of deaths in both HIV positive and negative patients. ${ }^{3}$ High levels of drug resistance among our chronic TB patients represents a risk for likely transmission of resistant strains to susceptible persons in the community as well as an increased mortality on standard drug therapy. A 20002004 survey of primary anti-TB drug resistance in Ghana on 2,064 isolates, reported then a high level of initial Streptomycin resistance of $23 \%$ in treatmentnaïve patients. ${ }^{17}$ The even higher prevalence of resistance to streptomycin in the present study $(79 \%)$ means the recommended WHO retreatment regimen which is routinely given in the absence of DST will not be effective and may even create greater resistance to the essential drugs in use as it fails to add on any effective new drug to the Category I regimen. Among the 28 positive cultures drug resistant TB was present in all groups irrespective of whether they had had one, two or three retreatments. This was particularly so with streptomycin and isoniazid, while rifampicin and MDR resistance appeared to be more common in patients who had completed three retreatments. We could not establish the significance of this trend given the small numbers of patients studied.

However the observed trend is what one would expect from the natural history of resistance development, ${ }^{15}$ and has been demonstrated in a large cohort in Uganda.

${ }^{18}$ Some authors have reported that even in the settings of moderate rates of MDR-TB, DOTS using only firstline drugs can be effective and reduce the transmission of both drug-susceptible and drug-resistant $\mathrm{TB}^{2}{ }^{2}$ but others have found DOTS alone to be inadequate in patients with drug-resistant TB. ${ }^{1,14,19}$ Urgent consideration is needed for the use of a standardized Category IV regimen combining effective first line drugs with selected second line drugs (including an injectable) for MDR, present in over a third of our chronic TB patients.

This has been feasible in other resource-limited countries with an efficient NTP, and has been demonstrated to be cost effective in Peru ${ }^{9,20}$ A major limitation of this study is the small number of chronic TB cases included in this report. Thus, the results should be interpreted with caution. This was a new laboratory service and selection bias could have occurred as some practitioners may not have been aware of the availability of mycobacterial culture or just did not have their chronic patients' sputum cultured in spite of repeated failures. The results of this review suggest that more studies on $M$. tuberculosis resistance are required, which will give support to the strengthening of the National TB Program, for application to the Green Light Committee for second line drugs.

\section{REFERENCES}

1. Espinal MA, Kim SJ, Suarez PG, Kam KM, Khomenko AG, Migliori GB et al. Standard shortcourse chemotherapy for drug-resistant tuberculsosis: treatment outcome in 6 countries. JAMA 2000; 283:2537-2545

2. DeRiemer K, GarcÃa-Garc̃̃a L, Bobadilla-delValle M, Palacios-MartÃnez M, MartÃnezGamboa A, Small PM, et al. Does DOTS work in populations with drug-resistant tuberculosis. Lancet 2005; 365:1239-1245

3. Kawai V, Soto G, Gilman R. H. Tuberculosis Mortality, Drug Resistance, And Infectiousness In Patients With And Without HIV Infection In Peru. Am. J. Trop. Med. Hyg 2006; 75(6): 1027-1033

4. Thwaites GE, Lan NT, Dung NH, Quy HT, Oanh DT, Thoa NT, et al. Effect of antituberculosis drug resistance on response to treatment and outcome in adults with tuberculosis meningitis. $J$ infect Dis 2005; 192:79-88 
5. Seung KJ, Gelmanova IE, Peremitin GG, Golubchikova VT, Pavlova VE, Sirotkina, et al. The effect of initial drug resistance on treatment response and acquired drug resistance during standardized short-course chemotherapy for tuberculosis. Clin Infect Dis 2004; 39:1321-1328.

6. Lockman S, Kruuner A, Binkin NJ, Levina K, Wang YC, Danilovitsh M, et al. Clinical outcomes of Estonian patients with primary multidrugresistant versus drug-susceptible tuberculosis. Clin Infect Dis 2001; 32:373-380

7. Zignol M, Hosseini MS, Wright A, Lambregtsvan Weezenbeek C, Nunn P, Watt CJ, et al. Global incidence of multidrug-resistant tuberculosis. $J$ Infect Dis 2006; 194:479-485

8. WHO. Treatment of tuberculosis: guidelines for national programmes. WHO/CDS/TB 2003.313

9. WHO. Guidelines for the programmatic management of drug-resistant tuberculosis. Geneva WHO/HTM/TB/2006.361

10. WHO. Anti-tbuerculosis drug resistance in the world: fourth global report. Geneva WHO/HTM/TB/2008.394.

11. The Public Health Service National Tuberculosis Reference Laboratory and National Laboratory Network. Minimum requirement, role and operation in a low income country. International Union Against TB and Lung Disease 1998.

12. Mycobacterium. In Color Atlas and textbook of Diagnostic Microbiology. Ed. Koneman E W, Allen S D, Dowell V R et al., 3rd Ed 1988: 544546

13. Canetti G, Fox W, Khomenko A. Advances in techniques of testing mycobacterial drug sensitivity and use of sensitivity tests in tuberculosis control programmes. Bull Wld Hlth Org 1969; 41:2143
14. Global tuberculosis control - surveillance, planning, financing. WHO Report 2008. WHO/

HTM/ TB/ 2008.393, ww.who.int/tb/publications/ global_report $/ 2008$

15. Gillespie S H. Evolution of Drug Resistance in Mycobacterium tuberculosis: Clinical and Molecular Perspective. Antimicrobial Agents and Chemotherapy 2002: 46 (2): 267-274

16. Newman MJ, Addo KK, Aboagye S, Bonsu FA, Caulley P, Hesse IF et al. Culture and sensitivity of mycobacterial isolates from cases of pulmonary tuberculosis classified as treatment failures in a teaching hospital. West Afr J Med. 2007 AprJun;26(2):131-133

17. Owusu-Dabo E, Adjei O, Meyer CG, Horstmann RD, Enimil A, Kruppa TF, et al. Mycobacterium tuberculosis drug resistance in Ghana. [letter]. Emerg Infect Dis. 2006 Jul http://www.cdc.gov/ncidod/EID/vol12no07/051028.htm

18. Temple B, Ayakaka I, Ogwang S, Nabanjja H, Kayes S, Nakubulwa S,et al. Rate and amplification of drug resistance among previously-treated patients with tuberculosis in Kampala, Uganda. Clin Infect Dis. 2008 Nov 1;47(9):1126-1134

19. Quy H T W, Lan N T N, Borgdorff M W, Grosset J, Linh P D, Tung L B, et al. Drug resistance among failure and relapse cases of tuberculosis: is the standard re-treatment regimen adequate? Int $J$ Tuber Lung Dis 2003; 7:631-636.

20. Suárez PG, Floyd K, Portocarrero J, Alarcón E, Rapiti E, Ramos G, et al. Feasibility and costeffectiveness of standardised second-line drug treatment for chronic tuberculosis patients: a national cohort study in Peru. Lancet, 2002, 359(9322): 1980-1989. 